\title{
PERÚ: DETERMINACIÓN DE LA BRECHA DE RECAUDACIÓN POTENCIAL DE LOS IMPUESTOS Y ARBITRIOS MUNICIPALES, 2014-2021
}

\author{
PERU: DETERMINATION OF THE POTENTIAL COLLECTION GAP FOR CITY TAXES, \\ 2014-2021
}

Luis EnRIQue Cayatopa Rivera Universidad Católica Sedes Sapientiae Lima, Perú

ORCID: https://orcid.org/0000-0002-6359-2125

Correo electrónico: luis.enrique.cayatopa.rivera@outlook.es

\section{RESUMEN}

Objetivo: Determinar las brechas sobre la recaudación potencial a largo plazo de cada tributo. Método: La investigación se abordó desde un enfoque cuantitativo, respecto al análisis de las series de tiempo de los impuestos y arbitrios municipales, desde enero 2014 hasta abril 2021. Complementariamente, se utilizó el filtro de Hodrick-Prescott. Resultados: En el periodo global de análisis, la diferencia entre la recaudación desestacionalizada y la recaudación potencial -tendencia - fue de 1237 millones de soles, con una contracción - brecha de recaudación - total de los tributos analizados en 2020 de 24,9 millones de soles. En tanto, entre 2019 y 2020 la recaudación nominal absoluta de los tributos municipales se redujo en 984,9 millones de soles, sustentados con la contracción individual de los siguientes tributos: predial, arbitrios, alcabala, al patrimonio vehicular, a los juegos de máquinas tragamonedas y a espectáculos públicos (no deportivos) con 13,66\%, 16,97\%, $35,11 \%, 20,26 \%, 69,96 \%$ y $82,62 \%$, respectivamente. Conclusión: Entre 2014 y abril de 2021 se presenta una tendencia alcista de largo plazo en la recaudación de los impuestos y arbitrios municipales, con contracciones cíclicas bajistas como la presentada en 2020 a efectos del coronavirus.

Palabras clave: Arbitrios; impuestos municipales; brecha de recaudación tributaria; gestión tributaria; COVID-19.
[Recibido: 08/06/2021 Aceptado: 23/08/2021 Publicado: 31/08/2021]

\begin{abstract}
Objective: Determine the gaps in the potential long-term collection of each tax. Method: The research was approached from a quantitative approach, regarding the analysis of the time series of property and city taxes, from January 2014 to April 2021. In addition, the Hodrick-Prescott filter was used. Results: In the global period of analysis, the difference between the seasonally adjusted collection and the potential collection -trend- was 1237 million soles, with a contraction -collection gap- total of the taxes analyzed in 2020 of 24.9 million soles. Meanwhile, between 2019 and 2020, the absolute nominal collection of taxes fell by 984.9 million soles, supported by the individual contraction of the following: property tax, city taxes, alcabala tax, vehicle tax, slot machine games tax and the tax on non-sporting public shows of $13.66 \%$; $16.97 \%$; $35.11 \%$; $20.26 \%$; $69.96 \%$ and $82.62 \%$ respectively. Conclusion: Between 2014 and April 2021 there is a long-term upward trend in the collection of municipal taxes and duties, with downward cyclical contractions such as the one presented in 2020 as consequence of the coronavirus.
\end{abstract}

Keywords: City taxes; property tax; tax collection gap; tax management; COVID-19. 


\section{INTRODUCCIÓN}

En virtud a los estudios empíricos, se evidencia una mayor proporción de documentos sobre el derecho tributario respecto de investigaciones que acotan el análisis estadístico subyacente sobre los ingresos municipales y, más limitado aún, sobre el análisis de los ciclos de recaudación municipal. Entre los papers que abordan el problema económico de la recaudación local, cabe mencionar a Huanqui y Narrea (2018), quienes desarrollan un análisis exploratorio sobre el problema secundante en la generación de ingresos propios, con especial énfasis en el impuesto predial, dada la dependencia de las transferencias del Gobierno central. Sobre el particular, recomiendan la actualización de los sistemas catastrales, así como una mejora en la gestión tributaria mediante la delegación de facultades a organismos autónomos como los Servicios de Administración Tributaria (SAT).

En otro estudio, Ruiz Vásquez (2017) aborda como investigación la recaudación de arbitrios municipales en el distrito de Lince entre los periodos 2011-2014, verificando un incremento de la morosidad desde $29,3 \%$ al 38,2\% en los extremos de análisis temporal, la misma que relaciona al bajo nivel de la cultura tributaria y la deficiente calidad de la gestión municipal.

Por su parte, Morales Gonzales (2009), en su estudio sobre la recaudación municipal en el Perú, refiere como una principal conclusión que "el desempeño del impuesto predial [...] es bastante heterogéneo, [acotando que las municipalidades ubicadas] en la costa recaudan en promedio casi diez veces más que las ubicadas en la sierra, y casi cinco que las localizadas en la selva” (p. 38).

En tanto, y a fin de comprender el marco legal que ampara la tributación municipal en el Perú, esta está normada por el Decreto Legislativo $N^{\circ} 776$ (1993) y sus modificatorias. En su artículo 6, refiere que los impuestos de orden municipal son los siguientes: predial, de alcabala, al patrimonio automotriz (vehicular), a las apuestas, a los juegos, y a los espectáculos públicos (no deportivos) (EPND).

Respecto al impuesto a los juegos de casino y máquinas tragamonedas, este está regulado por la Ley $\mathrm{N}^{\circ} 27153$ (1999) y sus modificatorias. Cabe acotar que, la principal característica de los impuestos es que el "[...] cumplimiento [de estos] no origina una contraprestación directa por parte de la Municipalidad al contribuyente" (Decreto Legislativo $\mathrm{N}^{\circ} 776,1993$, Art. 5). Adicionalmente, sobre los arbitrios municipales se menciona que "son las tasas que se paga por la prestación o mantenimiento de un servicio público individualizado en el contribuyente" (Decreto Legislativo $\mathrm{N}^{\circ} 776,1993$, Art. 68, lit. a).
Asimismo, las municipalidades ejecutan los ciclos de ingresos y gastos, regulados por el Decreto Legislativo $\mathrm{N}^{\circ}$ 1440 (2018) sobre el Sistema Nacional de Presupuesto Público y dispositivos vinculantes. En ese sentido, al ejecutar el ciclo de ingresos, los gobiernos locales registran a toda fuente de financiamiento, los ingresos determinados y recaudados, en el aplicativo informático Sistema Integrado de Administración Financiera (SIAF). Cabe mencionar que, como un subconjunto de registros Figuran los tributos en análisis.

Ahora bien, sobre el análisis coyuntural de 2020, la economía mundial se contrajo un 3,3\%, América Latina y El Caribe lo hizo en 7\%, y la economía peruana lo propio en $11,1 \%$ respecto a su PBI real (Fondo Monetario Internacional, 2021). Sobre el particular, una de las causas subyacentes de principal orden se vincula a las medidas restrictivas sobre la movilidad de las personas y la actividad económica que adoptaron los gobiernos para evitar la propagación del COVID-19.

En el caso de Perú, el 11 de marzo de 2020 el Gobierno declaró la emergencia sanitaria a nivel nacional mediante el Decreto Supremo No 008-SA (2020). En tanto, el 15 de marzo del mismo año se publicó el Decreto Supremo $\mathrm{N}^{\circ}$ 044-PCM (2020), mediante el cual se declaró el estado de emergencia nacional, decretándose la suspensión de algunos derechos constitucionales, la limitación al ejercicio de tránsito de las personas (cuarentena), salvo en algunas actividades específicas como el abastecimiento de suministros o los vinculados a la salud. También se restringieron las actividades comerciales, culturales, de transporte, recreativas, entre otras. En paralelo, el Gobierno peruano adoptó diversas estrategias a fin de mitigar las consecuencias de las medidas ejecutadas en el marco de la coyuntura sanitaria, tales como la transferencia de recursos a los gobiernos locales para paliar los gastos operativos como los servicios de saneamiento rural, seguridad ciudadana, gestión de residuos sólidos; así como modificar sus presupuestos por la menor recaudación tributaria, la entrega de canastas, la entrega de bonos a las personas vulnerables, entre otros.

Las medidas restrictivas adoptadas impactaron en varias aristas, entre las cuales sobresalen las vinculadas a la salud, educación, el empleo y también los niveles de recaudación de los gobiernos subnacionales. En esa misma línea, Pereyra Colchado (2020) advierte que la caída de la recaudación repercute directamente en la prestación de los servicios municipales como la limpieza pública, entre otros.

En tanto, como respuesta a la contracción de la recaudación por el COVID-19, varios países de Latinoamérica han optado por la ejecución de amnistías tributarias. Ante lo referido, Reyes-Tagle y Ospina (2020) acotan que 
las amnistías debieran de ser una excepción y no la regla, evaluando sobre el particular el costo neto y el riesgo vinculado. Así como diseñar las medidas focalizando el tipo de amnistías y el contribuyente objetivo, contemplando medidas complementarias como acuerdos o fraccionamientos de pago, y finalmente fortalecer el sistema de administración tributaria.

Por su parte, Larios y Melgarejo (2021) analizan las oportunidades para desarrollar nuevas herramientas para la proyección de ingresos tributarios en el marco del contexto COVID-19, proponiendo el uso de data analytics para permitir desarrollar modelos de recaudación tributaria que incorporen datos microeconómicos sensibles a las variables de la coyuntura sanitaria.

En virtud de lo referido, la presente investigación acota la siguiente pregunta: ¿Cuál ha sido el comportamiento histórico, en términos nominales y potenciales, de la recaudación de los tributos municipales en el Perú entre 2014 y el primer cuatrimestre de 2021? En la misma línea, se desarrollan los siguientes objetivos: calcular la variación de los ingresos municipales sobre la recaudación tributaria nominal; y determinar las brechas sobre la recaudación potencial de largo plazo de los principales tributos; haciendo un especial énfasis, para ambos objetivos, en el periodo del estado de emergencia por el COVID-19. A tal efecto, la hipótesis de investigación señala que, entre el 2014 y abril de 2021 la recaudación potencial de los tributos municipales presenta una tendencia alcista de largo plazo.

\section{MATERIAL Y MÉTODOS}

La investigación se abordó desde un enfoque cuantitativo, respecto al análisis de las series de tiempo de los impuestos y arbitrios municipales a nivel nacional, desde enero de 2014 hasta abril de 2021. A tal efecto, la fuente de información ha sido la Plataforma Nacional de Datos Abiertos de la Presidencia del Consejo de Ministros (2021).

A fin de determinar la tendencia (recaudación potencial) y el ciclo (brecha de la recaudación potencial) de los tributos analizados, se utilizó el filtro de Hodrick-Prescott (1981). En esa línea, en primer lugar, se analizó la descomposición de las series de tiempo según la ecuación (1).

$$
y_{t}=g_{t}+c_{t} \ldots(1)
$$

Donde: $g_{t}$ acota el componente tendencial, y $c_{t}$ el componente cíclico, para $t=1,2,3, \ldots, T$

Seguidamente, en el marco restrictivo de la ecuación (1), se aplicó el filtro Hodrick-Prescott (HP) en virtud de la siguiente operación de optimización:

$$
\min _{\left\{g_{t}\right\}_{t=-1}^{T}}\left\{\sum_{t=1}^{T} C_{t}^{2}+\lambda \sum_{t=1}^{T}\left[\left(g_{t}-g_{t-1}\right)-\left(g_{t-1}-g_{t-2}\right)\right]^{2}\right\} \ldots
$$

Donde $\lambda$ representa una medida del grado de suavidad del componente tendencial. En ese mismo hilo conductor, los autores recomendaron utilizar $\lambda=100$ para series anuales, $\lambda=1600$ para trimestres, y $\lambda=14400$ para análisis mensuales.

Adicionalmente, tras desestacionalizar la serie $y_{t}-y_{t}^{E}$ y calcular la tendencia $y_{t}^{T}$, se pudo determinar el componente cíclico a partir de la siguiente ecuación:

$$
y_{t}^{C}=y_{t}-y_{t}^{T}-y_{t}^{E} \ldots
$$

Sobre el particular, en la presente investigación se aplicó el filtro de Hodrick-Prescott al logaritmo de las series mensuales desestacionalizadas, mediante el método de ajuste de Tramo-SEATS de los 4 tributos de mayor recaudación.

En otro punto de análisis, se calcularon los indicadores de desempeño en términos relativos sobre los datos nominales, a fin de cuantificar las variaciones en el ingreso, respecto de las medidas restrictivas por la emergencia sanitaria.

\section{RESULTADOS}

\section{Contracciones sobre la recaudación nominal}

Las medidas adoptadas en el marco de la pandemia por la COVID-19, entre marzo y diciembre, han impactado en la recaudación municipal al cierre de 2020. En efecto, se evidencia una contracción del $21,48 \%$ en la recaudación acumulada de arbitrios e impuestos municipales respecto al periodo 2019, pasando de una recaudación nacional de 4,5 mil millones en 2019 a 3,6 mil millones de soles en 2020. De manera agrupada, la recaudación por arbitrios cerró el ejercicio 2020 con un 16,97\% de menores ingresos respecto al año anterior. Por su parte, la recaudación a todo nivel de impuestos municipales cerró a la baja en 2020 con un $23,29 \%$ de menores ingresos comparado con 2019 (Ver Tabla 1).

Cabe acotar que, a nivel individual, a excepción del impuesto a las apuestas, los demás tributos han cerrado a la baja en 2020. Así, el impuesto predial, que en términos nominales tiene mayor representatividad en la recaudación municipal, se contrajo a nivel nacional en $13,66 \%$ respecto a 2019, registrando una recaudación similar a la de 2018 . Por su parte, las restricciones sobre la actividad económica en el país impactaron en una relación directa con la disminución de los ingresos vinculados a los siguientes impuestos: de alcabala, vehicular, a los juegos de máquinas tragamonedas, y a los EPND principalmente, con contracciones de $35,11 \% ; 20,26 \% ; 69,96 \%$ y $82,62 \%$, respectivamente. Por su parte, las tasas vinculadas a la limpieza pública, serenazgo, y parques y jardines también registraron retrocesos en el orden de 19,63\%; 13,37\% y 15,13\%, 
respectivamente, lo que conllevó a la desestabilización de la prestación de los servicios municipales.

En un análisis pormenorizado, como se observa en la Figura 1 , la recaudación histórica del impuesto predial presentó picos históricos anuales al alza entre 2014 y febrero de 2020, con una clara estacionalidad en el segundo mes de cada año, pasando de 251,9 millones de soles en febrero de 2014 a 449,8 millones en febrero de 2020. Dos meses después, en abril, la recaudación nacional cayó al mínimo histórico de 12,3 millones de soles. En lo que va del periodo de emergencia sanitaria, en febrero 2021 se presentó la mayor recaudación en el orden de $350 \mathrm{mi}$ llones de soles, importe solo comparable al máximo registrado en febrero de 2017. Se advierte, asimismo, que en la región Lima, donde se concentra el 59,30\% de la recaudación nacional, la contracción al cierre de 2020 ha sido $9,13 \%$ respecto al 2019 , seguido por La Libertad con un ajuste a la baja del $17 \%$.

Tabla 1

Perú, recaudación tributaria. Arbitrios e impuestos municipales 2018-2020 (Expresado en soles)

\begin{tabular}{|c|c|c|c|c|}
\hline TRIBUTO MUNICIPAL & 2018 & 2019 & 2020 & Var $\%$ 20/19 \\
\hline ARBITRIOS MUNICIPALES & 1160088807,58 & 1313128165,51 & 1090252330,10 & $-16,97 \%$ \\
\hline LIMPIEZA PÚBLICA & 596739627,73 & 682030902,36 & 548122043,01 & $-19,63 \%$ \\
\hline SERENAZGO & 333014910,55 & 370796423,57 & 321222932,58 & $-13,37 \%$ \\
\hline PARQUES Y JARDINES & 230334269,30 & 260300839,58 & 220907354,51 & $-15,13 \%$ \\
\hline IMPUESTOS MUNICIPALES & 3014310012,26 & 3271800461,12 & 2509772161,31 & $-23,29 \%$ \\
\hline PREDIAL & 1746594272,18 & 2023997233,01 & 1747498234,44 & $-13,66 \%$ \\
\hline ALCABALA & 730877540,83 & 695695437,41 & 451443691,02 & $-35,11 \%$ \\
\hline AL PATRIMONIO VEHICULAR & 294006661,72 & 301988545,41 & 240819461,26 & $-20,26 \%$ \\
\hline A LOS JUEGOS DE MAQUINAS TRAGAMONEDAS & 176058477,43 & 178818603,53 & 53724118,53 & $-69,96 \%$ \\
\hline A LOS ESPECTACULOS PUBLICOS NO DEPORTIVOS & 44643129,15 & 49967622,24 & 8683377,60 & $-82,62 \%$ \\
\hline A LOS JUEGOS & 9537765,58 & 12929543,04 & 4774066,69 & $-63,08 \%$ \\
\hline A LOS CASINOS DE JUEGOS & 12354241,04 & 8364878,84 & 2754697,23 & $-67,07 \%$ \\
\hline A LAS APUESTAS & 237924,33 & 38597,64 & 74514,54 & $93,05 \%$ \\
\hline Total general & 4174398819,84 & 4584928626,63 & 3600024491,41 & $-21,48 \%$ \\
\hline
\end{tabular}

Fuente: Elaboración propia con base en los datos de la Presidencia del Consejo de Ministros (2021)

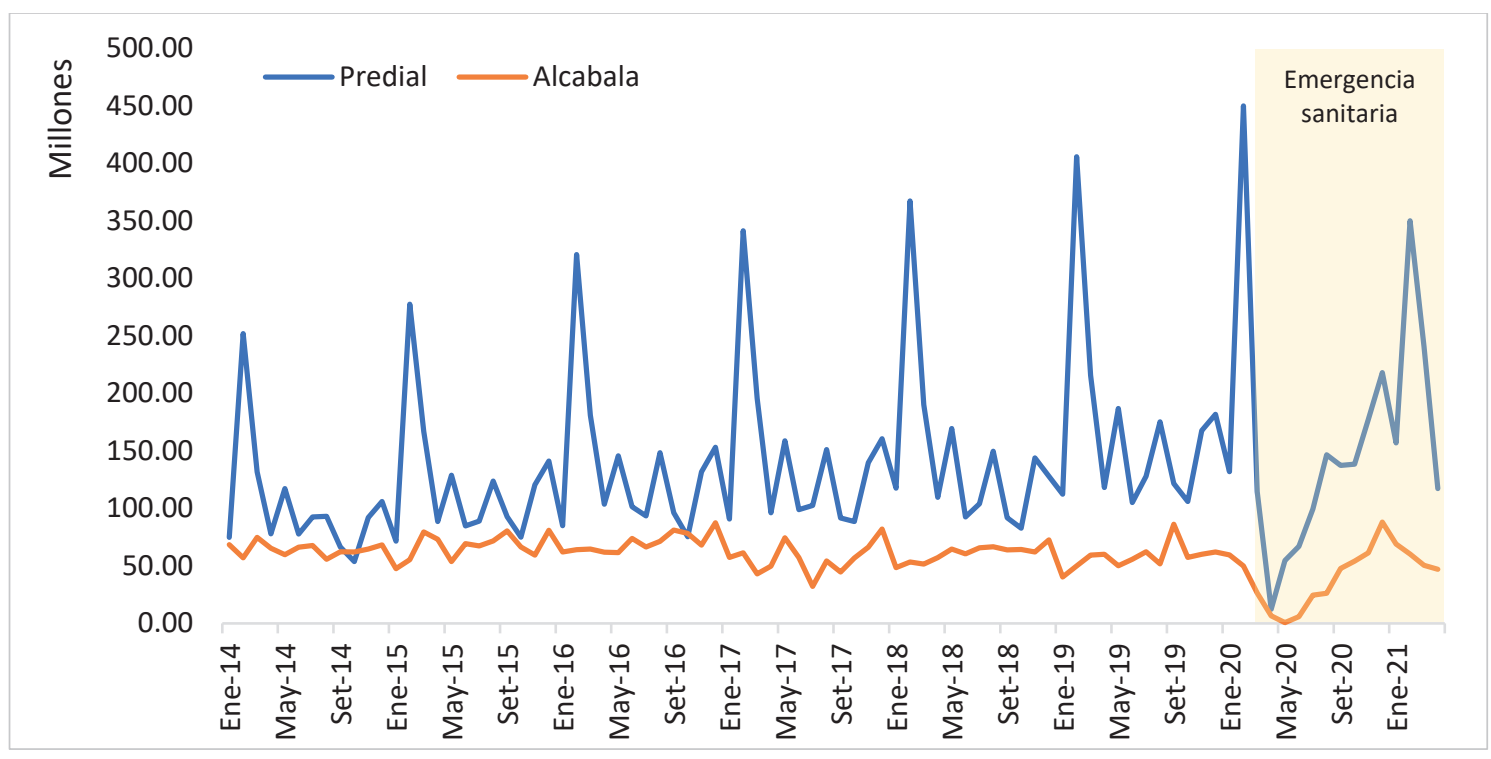

Figura 1. Perú, recaudación municipal. Impuestos: predial y alcabala de Ene 2014-Abr 2021 (Expresado en millones de soles)

Fuente: Elaboración propia con base en los datos de la Presidencia del Consejo de Ministros (2021) 
Por su parte, la liquidación del impuesto de alcabala se ajustó a la baja en $35,11 \%$ al cierre de 2020 en comparación con el periodo anterior. Sobre este particular, en mayo de 2020 se registró un mínimo histórico de 714 mil soles, siendo este un punto de rebote hasta un máximo en lo que va del periodo de emergencia sanitaria; de 88 millones de soles en diciembre de 2020. En lo que va de 2021 , entre enero y abril, la recaudación nacional alcanzó los 226 millones de soles, $59,08 \%$ más que la registrada en el mismo periodo de 2020 y $8,05 \%$ más respecto del primer cuatrimestre de 2019, evidenciándose una ligera recuperación de corto plazo en la recaudación nominal, que ha ido de la mano con la apertura de las actividades económicas en el país. A nivel regional, en Lima, donde se concentra el $63,78 \%$ de la recaudación nacional, la contracción al cierre de 2020 ha sido 37,57\% respecto al 2019, seguido por Arequipa con un ajuste a la baja del 44,76\% (Ver Tabla 2).

Por su parte, como se observa en la Figura 2, la estacionalidad recaudatoria del impuesto al patrimonio vehicular, pronunciada por los picos de ingresos de los meses de febrero, viene presentando un comportamiento sinuoso entre 2014 y abril de 2021, con una recaudación máxima en febrero

Tabla 2

Perú, recaudación municipal. Impuestos: predial y alcabala, según principales regiones 2018-2020 (Expresado en soles)

\begin{tabular}{|c|c|c|c|c|c|}
\hline TRIBUTO / REGIÓN & 2018 & 2019 & 2020 & Part. \% 2020 & Var \% 20/19 \\
\hline PREDIAL & 1746594272,18 & 2023997233,01 & 1747498234,44 & $100,00 \%$ & $-13,66 \%$ \\
\hline LIMA & 996094956,29 & 1140464260,47 & 1036321285,36 & $59,30 \%$ & $-9,13 \%$ \\
\hline LA LIBERTAD & 88792176,47 & 96378739,94 & 79996765,83 & $4,58 \%$ & $-17,00 \%$ \\
\hline AREQUIPA & 89786508,10 & 105624100,63 & 77971753,77 & $4,46 \%$ & $-26,18 \%$ \\
\hline OTRAS REGIONES & 571920631,32 & 681530131,97 & 553208429,48 & $31,66 \%$ & $-18,83 \%$ \\
\hline ALCABALA & 730877540,83 & 695695437,41 & 451443691,02 & $100,00 \%$ & $-35,11 \%$ \\
\hline LIMA & 504739831,06 & 461241611,30 & 287947464,32 & $63,78 \%$ & $-37,57 \%$ \\
\hline AREQUIPA & 40979887,30 & 49315451,68 & 27240336,43 & $6,03 \%$ & $-44,76 \%$ \\
\hline CALLAO & 28857305,95 & 21397051,21 & 26312383,02 & $5,83 \%$ & $22,97 \%$ \\
\hline OTRAS REGIONES & 156300516,52 & 163741323,22 & 109943507,25 & $24,35 \%$ & $-32,86 \%$ \\
\hline Total general & 2477471813,01 & 2719692670,42 & 2198941925,46 & & $-19,15 \%$ \\
\hline
\end{tabular}

Fuente: Elaboración propia con base en los datos de la Presidencia del Consejo de Ministros (2021)

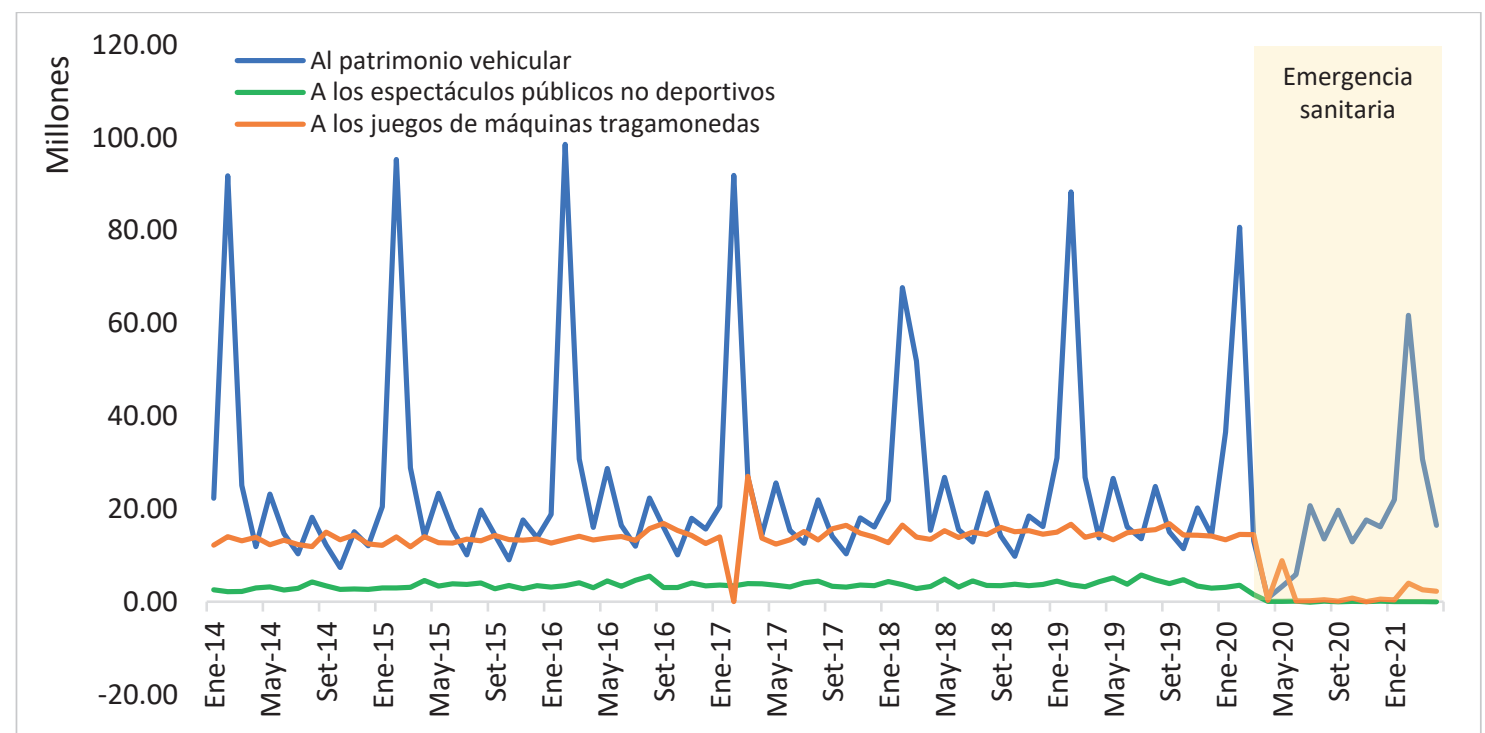

Figura 2. Perú, recaudación municipal. Impuestos: vehicular; espectáculos públicos no deportivos, y a los juegos de máquinas tragamonedas de Ene 2014-Abr 2021 (Expresado en millones de soles)

Fuente: Elaboración propia con base en los datos de la Presidencia del Consejo de Ministros (2021) 
de 2016, con un ingreso nominal nacional de 98,5 millones de soles y una contracción histórica sobre el máximo anual recaudado en febrero de 2021, registrando un total de 61,7 millones de soles. Cabe precisar que, la recaudación mínima mensual en el periodo de análisis se registró en abril de 2020 con 790 mil soles, coincidiendo con el periodo en el cual se adoptaron las restricciones normativas en el marco de la coyuntura sanitaria. En tanto, también se evidencia una alta concentración de la recaudación de este tributo en la región Lima con el 81,40\% de participación nacional en 2020, y cuya contracción por el efecto COVID-19 ha sido del orden de 16,25\% respecto de 2019.

Con relación a la recaudación de los impuestos sobre los juegos de máquinas tragamonedas y a los EPND, toda vez que están más vinculados al comportamiento de la actividad económica no presentan grados sustanciales de estacionalidad entre 2014 y abril de 2021; sin embargo, y como evidencian las estadísticas, sus niveles de contracción relativa por efectos de las medidas restrictivas en la economía en el marco de la coyuntura por el COVID-19, conllevaron a reducciones entre 2019 y 2020 , de $69,96 \%$ y $82,62 \%$, respectivamente.

Cabe precisar que ambos tributos presentan una concentración de recaudación en la región Lima, seguido de la región Ica en el caso de la recaudación del impuesto a los juegos de máquinas tragamonedas, y Arequipa en lo propio al impuesto a los EPND. En todos los casos, la contracción relativa de ingresos de estos impuestos a nivel regional, al cierre de 2020, supera el 60\% en comparación de 2019 (Ver Tabla 3).

En lo que respecta al análisis de las tasas de arbitrios municipales, en términos agregados, la recaudación -consolidado de limpieza pública, serenazgo, y parques y jardines - ha seguido el comportamiento alcista del impuesto predial entre enero de 2014 y febrero de 2020, pasando de 187,3 millones en febrero de 2014 a 323,05 millones de soles en febrero de 2020; mes en el cual se presentó un punto de inflexión a la baja, llegando a febrero de 2021 como máxima recaudación desde que se declaró el estado de emergencia, en el orden de 246,6 millones de soles,

Tabla 3

Perú, recaudación municipal. Impuestos: vehicular, espectáculos públicos no deportivos y a los juegos de máquinas tragamonedas, según regiones 2018-2020

(Expresado en soles)

\begin{tabular}{|c|c|c|c|c|c|}
\hline TRIBUTO / REGIÓN & 2018 & 2019 & 2020 & $\begin{array}{c}\text { Part. \% } \\
2020\end{array}$ & $\begin{array}{l}\text { Var \% } \\
20 / 19\end{array}$ \\
\hline AL PATRIMONIO VEHICULAR & 294006661,72 & 301988545,41 & 240819461,26 & $100,00 \%$ & $-20,26 \%$ \\
\hline LIMA & 231841400,35 & 234082372,07 & 196039002,34 & $81,40 \%$ & $-16,25 \%$ \\
\hline AREQUIPA & 17738273,17 & 18136300,03 & 10983894,01 & $4,56 \%$ & $-39,44 \%$ \\
\hline LA LIBERTAD & 8223699,53 & 8091273,73 & 5537363,49 & $2,30 \%$ & $-31,56 \%$ \\
\hline OTRAS REGIONES & 36203288,67 & 41678599,58 & 28259201,42 & $11,73 \%$ & $-32,20 \%$ \\
\hline A LOS JUEGOS DE MAQUINAS TRAGAMONEDAS & 176058477,43 & 178818603,53 & 53724118,53 & $100,00 \%$ & $-69,96 \%$ \\
\hline LIMA & 114478878,57 & 118685316,51 & 36123926,87 & $67,24 \%$ & $-69,56 \%$ \\
\hline ICA & 5672283,09 & 6026051,66 & 2170346,35 & $4,04 \%$ & $-63,98 \%$ \\
\hline AREQUIPA & 6460960,44 & 6530229,94 & 1932056,65 & $3,60 \%$ & $-70,41 \%$ \\
\hline OTRAS REGIONES & 49446355,33 & 47577005,42 & 13497788,66 & $25,12 \%$ & $-71,63 \%$ \\
\hline A LOS ESPECTACULOS PUBLICOS NO DEPORTIVOS & 44643129,15 & 49967622,24 & 8683377,60 & $100,00 \%$ & $-82,62 \%$ \\
\hline LIMA & 28899082,54 & 32841032,06 & 5438291,92 & $62,63 \%$ & $-83,44 \%$ \\
\hline AREQUIPA & 2060407,53 & 2283502,16 & 373573,44 & $4,30 \%$ & $-83,64 \%$ \\
\hline LA LIBERTAD & 2073718,24 & 2223205,85 & 336572,35 & $3,88 \%$ & $-84,86 \%$ \\
\hline OTRAS REGIONES & 11609920,84 & 12619882,17 & 2534939,89 & $29,19 \%$ & $-79,91 \%$ \\
\hline Total general & 514708268,30 & 530774771,18 & 303226957,39 & & $-42,87 \%$ \\
\hline
\end{tabular}

Fuente: Elaboración propia con base en los datos de la Presidencia del Consejo de Ministros (2021) 
importe comparable con lo registrado en febrero de 2017 (Ver Tabla 4).

Por su parte, en lo que va del primer cuatrimestre de 2021, la recaudación ha alcanzado los 588,7 millones de soles, $18,02 \%$ más que en el mismo periodo de 2020, y todavía $1,4 \%$ por debajo de lo reportado en el mismo periodo comparativo de 2019.
A nivel regional, se evidencia una fuerte concentración de la recaudación nacional de los arbitrios en la región Lima, con el 68,28\% sobre la limpieza pública, el 81,62\% respecto del serenazgo, y el $76,78 \%$ sobre parques y jardines. Cabe precisar que, existen regiones, en el caso de la recaudación por serenazgo, donde en ningún distrito se evidencia el ingreso vinculado. Tal es el caso histórico de la región Apurímac (Ver Figura 3).

Tabla 4

Perú, recaudación municipal. Arbitrios municipales 2018-2020 (Expresado en soles)

\begin{tabular}{|c|c|c|c|c|c|}
\hline TRIBUTO / REGIÓN & 2018 & 2019 & 2020 & Part. \% 2020 & Var \% 20/19 \\
\hline LIMPIEZA PUBLICA & 596739627,73 & 682030902,36 & 548122043,01 & $100,00 \%$ & $-19,63 \%$ \\
\hline LIMA & 401746875,28 & 457917380,57 & 374264872,01 & $68,28 \%$ & $-18,27 \%$ \\
\hline CALLAO & 42124126,29 & 50387394,21 & 48005775,31 & $8,76 \%$ & $-4,73 \%$ \\
\hline LAMBAYEQUE & 16545018,32 & 21404737,38 & 17236134,90 & $3,14 \%$ & $-19,48 \%$ \\
\hline OTRAS REGIONES & 136323607,84 & 152321390,20 & 108615260,79 & $19,82 \%$ & $-28,69 \%$ \\
\hline SERENAZGO & 333014910,55 & 370796423,57 & 321222932,58 & $100,00 \%$ & $-13,37 \%$ \\
\hline LIMA & 262754316,18 & 290897720,84 & 262172311,35 & $81,62 \%$ & $-9,87 \%$ \\
\hline CALLAO & 15354029,14 & 17181321,29 & 13935200,33 & $4,34 \%$ & $-18,89 \%$ \\
\hline LA LIBERTAD & 16117429,62 & 15752043,76 & 11872183,32 & $3,70 \%$ & $-24,63 \%$ \\
\hline OTRAS REGIONES & 38789135,61 & 46965337,68 & 33243237,58 & $10,35 \%$ & $-29,22 \%$ \\
\hline PARQUES Y JARDINES & 230334269,30 & 260300839,58 & 220907354,51 & $100,00 \%$ & $-15,13 \%$ \\
\hline LIMA & 170411999,10 & 193868661,12 & 169609988,39 & $76,78 \%$ & $-12,51 \%$ \\
\hline CALLAO & 14796364,56 & 17082912,60 & 16154334,66 & $7,31 \%$ & $-5,44 \%$ \\
\hline LA LIBERTAD & 15161784,93 & 14020924,54 & 9964377,78 & $4,51 \%$ & $-28,93 \%$ \\
\hline OTRAS REGIONES & 29964120,71 & 35328341,32 & 25178653,68 & $11,40 \%$ & $-28,73 \%$ \\
\hline Total general & 1160088807,58 & 1313128165,51 & 1090252330,10 & & $-16,97 \%$ \\
\hline
\end{tabular}

Fuente: Elaboración propia con base en los datos de la Presidencia del Consejo de Ministros (2021)

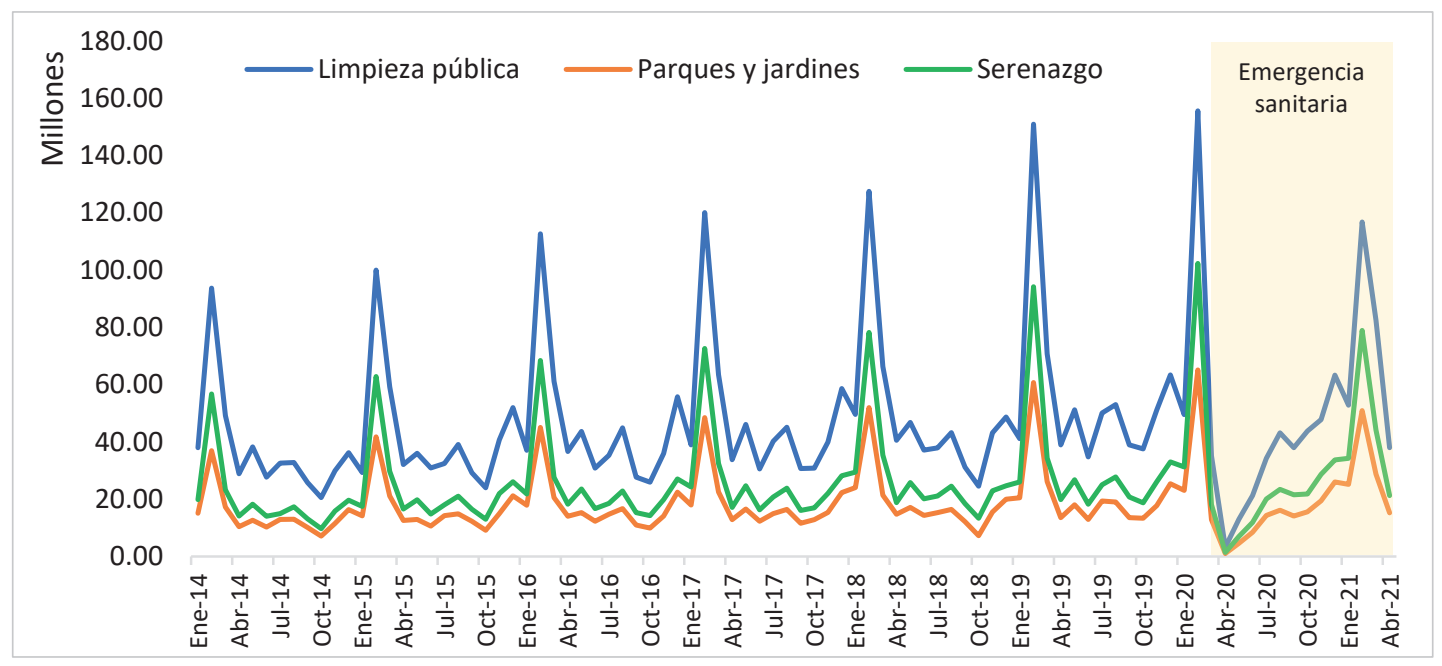

Figura 3. Perú, recaudación municipal. Arbitrios municipales de Ene 2014-Abr 2021

(Expresado en millones de soles)

Fuente: Elaboración propia con base en los datos de la Presidencia del Consejo de Ministros (2021) 


\section{Brechas sobre la recaudación potencial}

A nivel global, entre 2014 y abril de 2021, se observa que la diferencia entre la recaudación ajustada por factores de desestacionalización y la recaudación potencial de largo plazo es del orden de 1237 millones de soles, dato que confirmaría la tendencia alcista de las series de recaudación de los tributos analizados (Ver Tabla 5).

Asimismo, en los años 2014, 2017 y 2020 se observa una brecha sobre la recaudación de 66,5; 54,8 y 24,9 millones de soles, respectivamente, por debajo de la recaudación potencial de largo plazo. Cabe resaltar que, desde que se decretó el estado de emergencia nacional por la pandemia, la recaudación de todos los tributos analizados se contrajo, en especial entre el segundo y tercer trimestre de 2020.

En otro punto de análisis, a nivel pormenorizado, en primer lugar en virtud de los resultados sobre el impuesto predial, se evidencia una caída histórica mensual en la recaudación de abril de 2020, en el orden de 2,09\% respecto a la recaudación potencial a partir de las medidas de inmovilización. Asimismo, se observa que en los meses subsiguientes, conforme se fueron flexibilizando las medidas, los niveles de recaudación han venido recuperándose. Así, en enero de 2021 las municipalidades a nivel nacional recaudaron $0,42 \%$ más respecto a la tendencia de crecimiento de largo plazo; sin embargo, en febrero de 2021, mes en el cual se acrecientan los factores de estacionalidad, la recaudación se situó ligeramente por encima de la recaudación potencial $(0,02 \%)$.

Tabla 5

Perú, brechas de recaudación municipal. 2014-abril 2021 (Expresado en soles)

\begin{tabular}{|c|c|c|c|c|c|c|}
\hline FECHA & ARBITRIOS & ALCABALA & PREDIAL & TRAGAMONEADAS & VEHICULAR & BRECHA TOTAL \\
\hline 2014 & $-14,463,406.85$ & $-19,399,244.54$ & $-30,249,573.87$ & $1,927,770.86$ & $-4,336,459.14$ & $-66,520,913.54$ \\
\hline 2015 & $13,744,356.15$ & $6,041,610.41$ & $20,475,935.95$ & $1,552,422.72$ & $-1,674,125.88$ & $40,140,199.35$ \\
\hline 2016 & $-603,153.82$ & $57,748,624.03$ & $31,212,606.06$ & $9,508,309.17$ & $8,326,027.62$ & $106,192,413.07$ \\
\hline 2017 & $-1,190,018.10$ & $-50,600,200.09$ & $1,778,883.95$ & $1,393,929.96$ & $-6,253,380.91$ & $-54,870,785.18$ \\
\hline 2018 & $12,283,616.72$ & $75,713,335.28$ & $-8,299,151.23$ & $20,555,560.34$ & $11,409,470.74$ & $111,662,831.85$ \\
\hline 2019 & $209,773,589.13$ & $154,321,542.95$ & $288,111,789.70$ & $93,479,289.51$ & $51,144,076.41$ & $796,830,287.70$ \\
\hline 2020 & $-50,416,733.46$ & $307,071.43$ & $-24,821,332.53$ & $34,039,281.44$ & $15,974,184.65$ & $-24,917,528.46$ \\
\hline Ene & $24,351,376.65$ & $23,872,160.54$ & $43,470,386.23$ & $10,529,954.90$ & $17,288,169.32$ & $119,512,047.64$ \\
\hline Feb & $27,200,176.70$ & $15,136,052.32$ & $46,419,046.89$ & $11,434,907.50$ & $6,940,762.77$ & $107,130,946.17$ \\
\hline Mar & $-36,812,153.56$ & $-9,801,739.06$ & $-62,509,610.55$ & $12,790,599.53$ & $-7,916,597.52$ & $-104,249,501.16$ \\
\hline Abr & $-80,839,941.81$ & $-30,460,633.16$ & $-126,206,920.72$ & $-2,148,139.60$ & $-17,588,513.46$ & $-257,244,148.75$ \\
\hline May & $-61,247,872.59$ & $-36,382,833.49$ & $-102,361,520.38$ & $8,572,551.47$ & $-15,698,511.13$ & $-207,118,186.11$ \\
\hline Jun & $-22,569,014.44$ & $-30,900,340.00$ & $-41,912,567.14$ & $-1,736,796.34$ & $-9,910,974.91$ & $-107,029,692.82$ \\
\hline Jul & $-5,756,257.19$ & $-12,863,604.40$ & $-14,109,126.94$ & $-1,337,572.02$ & $17,094,442.86$ & $-16,972,117.69$ \\
\hline Ago & $3,322,440.69$ & $-9,327,187.99$ & $4,155,216.17$ & $-1,095,900.82$ & $-6,272,694.74$ & $-9,218,126.69$ \\
\hline Set & $18,847,342.49$ & $6,386,772.96$ & $39,003,408.95$ & $-1,125,663.10$ & $10,279,903.61$ & $73,391,764.90$ \\
\hline Oct & $31,288,480.84$ & $18,147,332.98$ & $61,296,885.92$ & $-487,214.62$ & $8,540,761.63$ & $118,786,246.75$ \\
\hline Nov & $24,744,250.08$ & $26,645,679.11$ & $53,281,885.36$ & $-968,077.09$ & $5,985,474.68$ & $109,689,212.13$ \\
\hline Dic & $27,054,438.69$ & $39,855,411.63$ & $74,651,583.68$ & $-389,368.37$ & $7,231,961.53$ & $148,404,027.16$ \\
\hline 2021 & $87,560,866.51$ & $102,527,878.67$ & $113,165,310.39$ & $6,233,848.27$ & $19,572,713.88$ & $329,060,617.72$ \\
\hline Total general & $256,689,116.30$ & $326,660,618.14$ & $391,374,468.43$ & $168,690,412.26$ & $94,162,507.38$ & $1,237,577,122.50$ \\
\hline
\end{tabular}

Nota: La brecha de recaudación se calcula de la diferencia de la serie de recaudación desestacionalizada menos la tendencia de recaudación de largo plazo.

Fuente: Elaboración propia con base en los datos de la Presidencia del Consejo de Ministros (2021) 
En tanto, del análisis de la recaudación potencial, se observa una recuperación al alza en la tendencia de mediano y largo plazo (Ver Figura 4).

En segundo lugar, sobre el comportamiento de los ingresos por el impuesto de alcabala, se observa una caída mensual en la recaudación de mayo de 2020 en el orden de 3,89\% respecto a la recaudación potencial. Asimismo, se observa que, en los siguientes meses, los niveles de recaudación han venido recuperándose relativamente; por ejemplo, en enero de 2021 las municipalidades, en promedio, recaudaron $0,8 \%$ más respecto a la tendencia de crecimiento de largo plazo. En tanto, sobre el análisis de la recaudación potencial entre 2014 y abril de 2021, se observa una ligera corrección del orden de $0,65 \%$ a la baja en la tendencia, a pesar de la recuperación nominal en la recaudación del primer cuatrimestre de 2021 acrecentada en el marco de la coyuntura sanitaria (Ver Figura 5). En tercer lugar, sobre el análisis del impuesto al patrimonio vehicular, se observó una caída en la recaudación de abril de 2020, en el orden de 2,7\% respecto a su tendencia. Por otra parte, sobre el análisis de la recaudación potencial entre 2014 y abril de 2021, se observa una ligera corrección del orden de $0,18 \%$ a la baja, acrecentada en

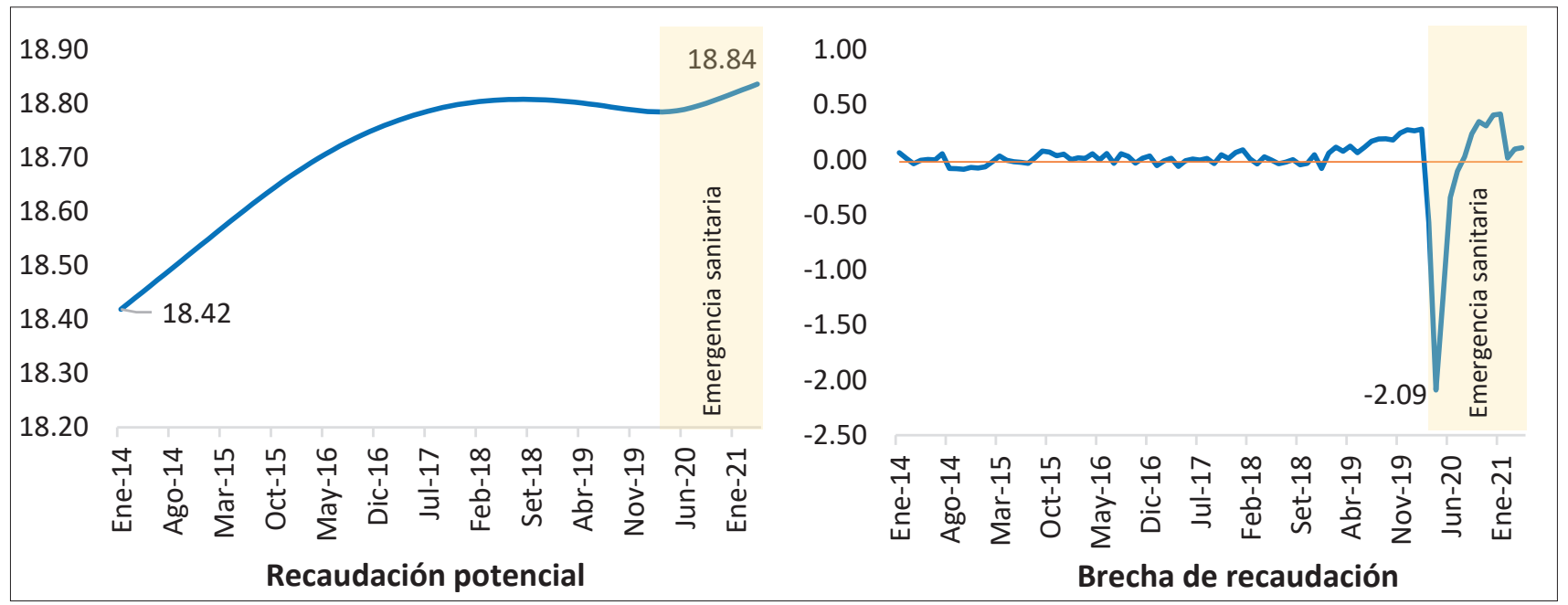

Figura 4. Impuesto predial. Comportamiento de la brecha sobre la recaudación potencial de 2014-Abr 2021 Fuente: Elaboración propia con base en los datos de la Presidencia del Consejo de Ministros (2021)

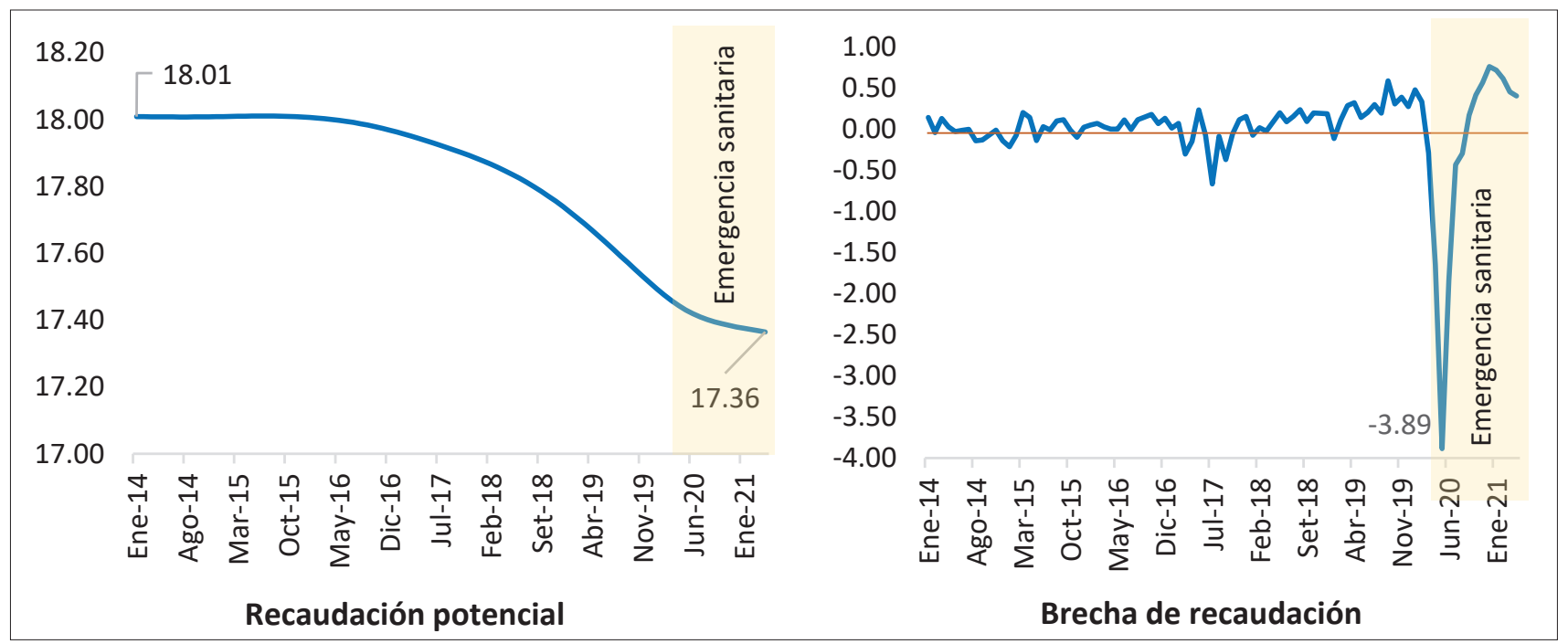

Figura 5. Impuesto de alcabala. Comportamiento de la brecha sobre la recaudación potencial de 2014-Abr 2021 Fuente: Elaboración propia con base en los datos de la Presidencia del Consejo de Ministros (2021) 
el marco de la coyuntura sanitaria, pero con una inflexión observada a la baja desde 2017. Sobre el particular, cabe acotar que la recaudación de este tributo está directamente relacionada con el comportamiento de la venta de vehículos nuevos en el mercado peruano (Ver Figura 6). Finalmente, sobre el comportamiento de los ingresos por arbitrios municipales, se observó una brecha de recaudación de 2,34\% en abril de 2020, vinculado entre otras causas a las medidas restrictivas adoptadas en pro de limitar la propagación del COVID-19. Por su parte, en los meses subsiguientes, conforme se fueron flexibilizando las medidas vinculadas a las limitaciones de las actividades económicas, los niveles de recaudación han venido recuperándose relativamente; por ejemplo, en enero de 2021 las municipalidades a nivel nacional recaudaron $0,3 \%$ más respecto a la tendencia. En tanto, sobre el análisis de la recaudación potencial entre 2014 y abril de 2021, se observa una ligera corrección alcista en la tendencia de largo plazo, con una contracción en la recaudación de los primeros meses de la coyuntura sanitaria. Cabe resaltar que, en 2020, como respuesta a las contracciones bajistas en la recaudación de inicios de la pandemia, el Gobierno transfirió recursos a las municipalidades a fin de subsidiar la continuidad de los servicios municipales afectados coyunturalmente (Ver Figura 7).

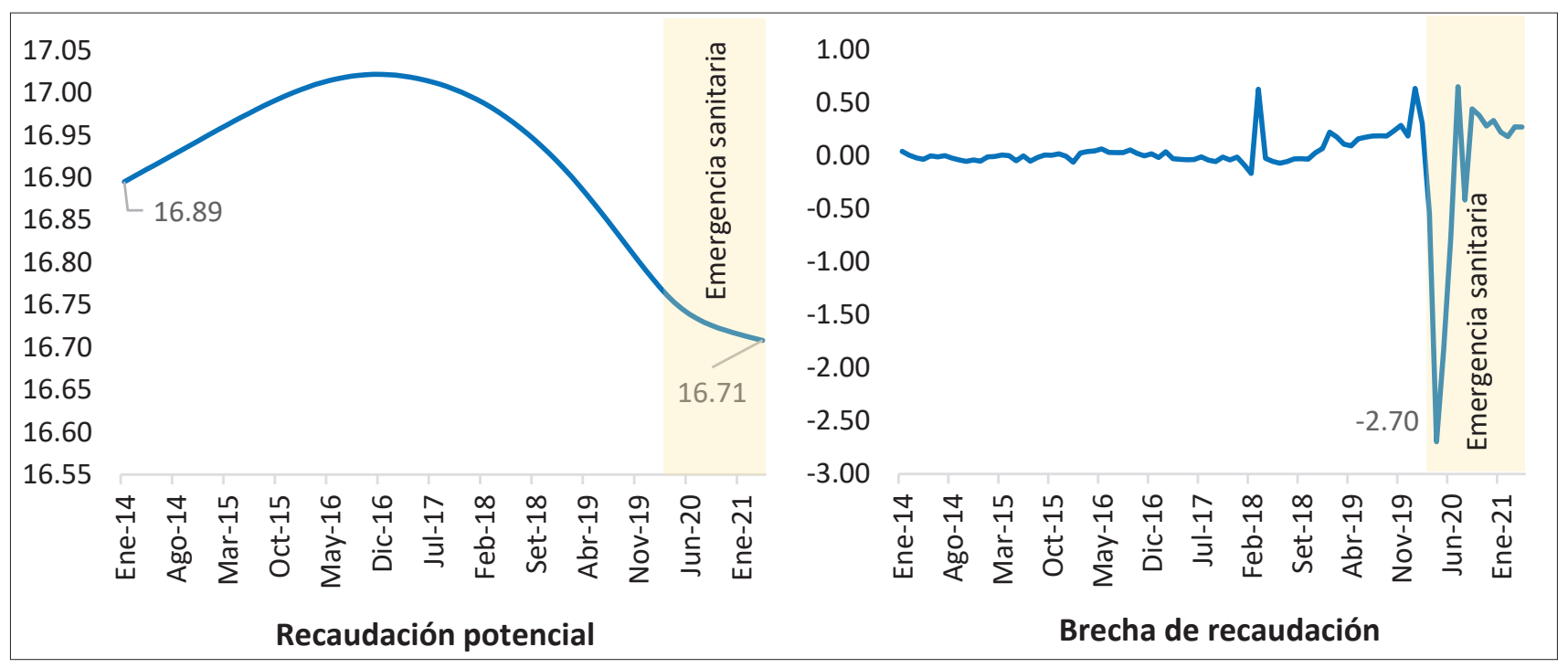

Figura 6. Impuesto al patrimonio vehicular. Comportamiento de la brecha sobre la recaudación potencial de 2014-Abr 2021 Fuente: Elaboración propia con base en los datos de la Presidencia del Consejo de Ministros (2021)

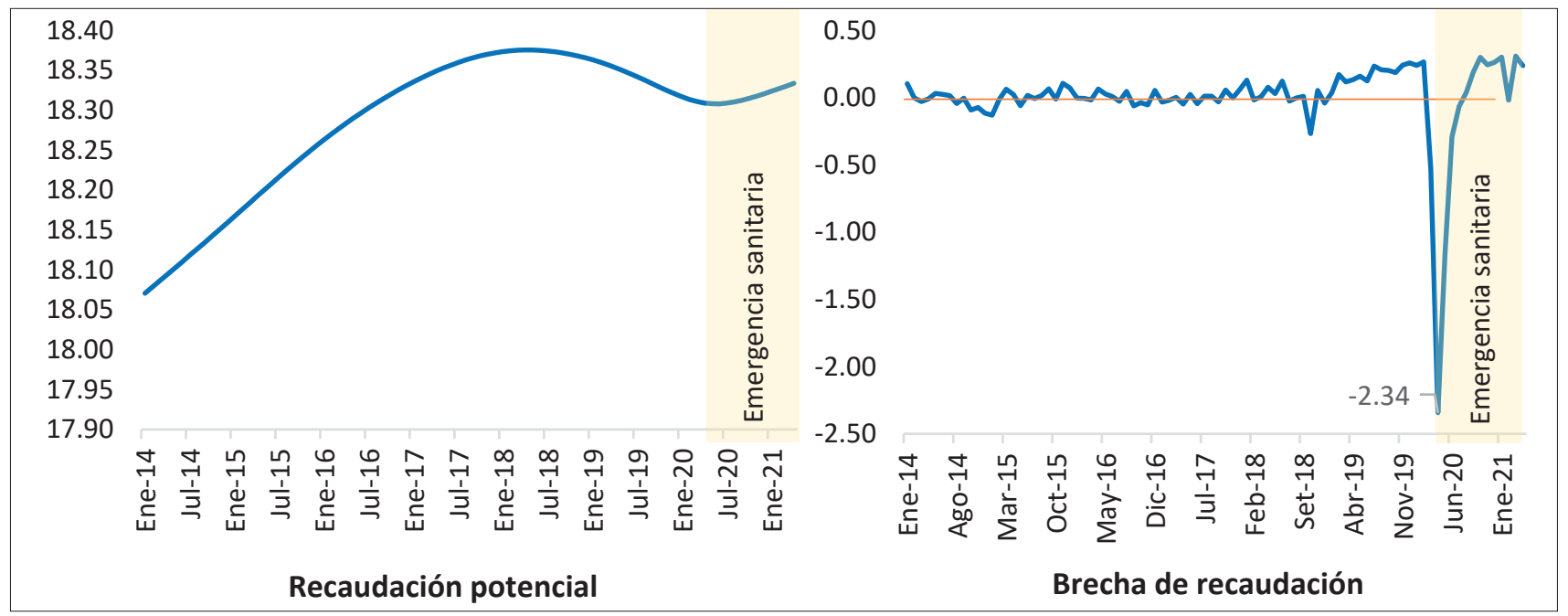

Figura 7. Arbitrios municipales. Comportamiento de la brecha sobre la recaudación potencial de 2014-Abr 2021

Fuente: Elaboración propia con base en los datos de la Presidencia del Consejo de Ministros (2021) 


\section{DISCUSIÓN}

A la luz de los datos analizados, se acepta la hipótesis de investigación, confirmándose la tendencia alcista de largo plazo de los tributos en referencia. Se acota que, entre 2014 y abril de 2021, la diferencia entre la recaudación municipal a nivel nacional, ajustada por factores estacionales y la recaudación esperada o potencial de largo plazo -tendencia-, ha sido del orden de 1237 millones de soles; observándose una contracción coyuntural en 2020 del orden de 24,9 millones de soles a raíz de las medidas restrictivas adoptadas en el marco de la pandemia por el COVID-19.

En términos nominales, al cierre de 2020 y respecto al 2019, se observó una disminución absoluta en la recaudación por arbitrios e impuestos municipales en el orden de 984,9 millones de soles. En esa línea, el impuesto predial se contrajo en un $13,66 \%$. Por su parte, los ingresos de los impuestos de alcabala, vehicular, a los juegos de máquinas tragamonedas y a los EPND cerraron a la baja en $35,11 \%$, $20,26 \%, 69,96 \%$ y $82,62 \%$, respectivamente. En tanto, a nivel nacional de forma conjunta, la recaudación de los arbitrios municipales se contrajo en $16,97 \%$, siendo la tasa por limpieza pública la que reportó una menor recaudación, con una variación relativa a la baja de 19,63\%.

En virtud de los resultados, se confirman de manera indirecta las conclusiones de Morales Gonzales (2009) sobre la heterogeneidad de la recaudación del impuesto predial, según región natural, con una concentración mayoritaria de los ingresos en las regiones costeñas de Lima, La Libertad y Arequipa principalmente, en la medida que la misma relación de heterogeneidad se extiende a los demás impuestos y arbitrios municipales.

Asimismo, vista la contracción nominal y la brecha coyuntural de 2020, reflejada en una menor recaudación, se infieren también mayores márgenes de morosidad a nivel local en los tributos analizados. Sobre el particular, impera un gran reto para las administraciones locales en fortalecer la cultura tributaria, considerando además que antes de la declaración del estado de emergencia por el COVID-19, ya los indicadores de morosidad rondaban la tercera parte de la base imponible a la luz las conclusiones del estudio puntual de Ruiz Vásquez (2017).

Ahora bien, en el marco del análisis coyuntural por la pandemia del coronavirus, se generaron múltiples desafíos para los diversos sistemas de salud en el mundo, y paralelamente la mayor parte de gobiernos adoptaron diversas medidas a fin de mitigar la propagación del virus. Sobre el particular, el Banco Mundial (2021) en su último informe acotó que se evidenciaron "confinamientos generalizados, cierres de escuelas y de empresas, y pérdidas de puestos de trabajo. [En tanto,] casi todos los países enfrentan [actualmente] una desaceleración económica sin precedentes" (p. 9), los mismos que a su vez repercutieron en el nivel de recaudación tributaria.

En esa misma línea de ideas, Perú no ha sido ajeno en promulgar dispositivos legales vinculados a inmovilizaciones obligatorias, tanto en la esfera personal como de las actividades económicas que coadyuvaron la declaratoria del estado de emergencia nacional, siendo una de las consecuencias de la coyuntura sanitaria las medidas restrictivas y la contracción del PBI real de Perú en el orden de 11,1\% al cierre de 2020 (Fondo Monetario Internacional, 2021).

Así, ante los datos expuestos y la coyuntura actual, al margen de la tendencia alcista de largo plazo de la recaudación municipal, y en línea con Larios y Melgarejo (2021), es preciso acotar que la crisis por el COVID-19 debería aprovecharse como una oportunidad de innovación para la gestión tributaria municipal. A tal efecto, sería importante el uso de data analytics a fin de desarrollar modelos que incorporen variables sensibles a la coyuntura, utilizando información microeconómica como por ejemplo: la Encuesta Nacional de Hogares (ENAHO) o datos de movilidad, así como pormenorizar el análisis de recaudación a nivel subnacional, toda vez que la evidencia demuestra que existen varias municipalidades que no recaudan los diversos tributos analizados; y en su defecto, la continuidad de la prestación de los diversos servicios públicos está siendo condicionado en virtud de las transferencias del Gobierno central.

De esta forma, sería importante fortalecer paulatina y de forma sostenible en el tiempo, los mecanismos de recaudación no presencial, incorporando como estrategia una meta vinculante en el Programa de Incentivos para la Mejora de la Gestión Municipal; y en paralelo, analizar el costo-beneficio de la delegación de facultades de la gestión tributaria a organismos autónomos como los Servicios de Administración tributaria en línea con lo planteado por Huanqui y Narrea (2018).

Finalmente, como agenda futura de investigación, es importante absolver las siguientes interrogantes: ¿Cuáles son las principales relaciones de causalidad entre las actividades económicas subyacentes y la recaudación municipal vinculantes, por ejemplo, la venta de vehículos nuevos, o la trasferencia de inmuebles, respecto de la recaudación del impuesto al patrimonio vehicular y el impuesto de alcabala, respectivamente? Asimismo, ¿qué tan sensible puede ser la recaudación municipal respecto al ciclo político peruano? 


\section{REFERENCIAS}

Banco Mundial. (2021). Informe anual 2020. Recuperado de: https://www.bancomundial.org/es/about/annual-report\#anchor-annual

Decreto Legislativo $\mathrm{N}^{\circ}$ 1440, Decreto Legislativo del Sistema Nacional de Presupuesto Publico. (2018). Poder Ejecutivo. Diario oficial El Peruano, 16 de setiembre

Decreto Legislativo $N^{\circ} 776$, Ley de tributación municipal. (1993). Poder Ejecutivo. Diario oficial El Peruano, 30 de diciembre.

Decreto Supremo No 008-SA, Decreto Supremo que declara en Emergencia Sanitaria a nivel nacional [...] y dicta medidas de prevención y control del Covid-19. (2020). Poder Ejecutivo. Diario oficial El Peruano, 11 de marzo.

Decreto Supremo $N^{\circ}$ 044-PCM, Decreto Supremo que declara Estado de Emergencia Nacional por las graves circunstancias que afectan la vida de la Nación a consecuencia del brote del Covid-19. (2020). Poder Ejecutivo. Diario oficial El Peruano, 15 de marzo.

Fondo Monetario Internacional. (2021). Informe de perspectivas de la economía mundial. Recuperado de: https://www.imf.org/es/Publications/WEO/Issues/2021/03/23/world-economic-outlook-april-2021

Huanqui, S., \& Narrea, O. (2018). Fortalecimiento de la autonomía fiscal de los gobiernos locales. El caso del impuesto predial. Recuperado de: https://www.up.edu. pe/egp/Documentos/Nota-de-Politica-N5.pdf

Hodrick, R. J., \& Prescott, E. (1981). Post-War U.S. Business Cycles: An Empirical Investigation. Discussion Papers 451, 1-24. Recuperado de: https://www.kellogg. northwestern.edu/research/math/papers/451.pdf.

Larios, J., \& Melgarejo, K. (30 de marzo de 2021). Recaudando Bienestar. La covid-19 presenta una oportunidad para desarrollar nuevas herramientas de proyecciones de ingresos: El Caso de Panamá. Recuperado de: https://blogs.iadb. org/gestion-fiscal/es/la-covid-19-presenta-una-oportunidad-para-desarrollar-nuevas-herramientas-de-proyecciones-de-ingresos-el-caso-de-panama/
Ley $\mathrm{N}^{\circ} 27153$, Ley que regula la explotación de los juegos de casino, máquinas tragamonedas. (1999). Congreso de la República. Diario Oficial El Peruano, 18 de junio.

Morales Gonzales, A. (2009). Los impuestos locales en el Perú: Aspectos institucionales y desempeño fiscal del impuesto predial. Recuperado de: https://cies.org.pe/ sites/default/files/investigaciones/el-rol-de-los-tributos-locales-en-el-financiamiento-municipal-el-caso-del-impuesto-predial.pdf

Pereyra Colchado, G. (27 de abril de 2020). Otro efecto de la cuarentena: la caída en recaudación de arbitrios y el riesgo en la limpieza pública. El Comercio. Recuperado de: https:/elcomercio.pe/lima/ sucesos/coronavirus-peru-otro-efecto-de-la-cuarentena-la-caida-en-recaudacion-de-arbitrios-y-el-riesgo-en-la-limpieza-publica-covid-19-noticia/?ref=ecr

Presidencia del Consejo de Ministros. (2021). Plataforma Nacional de datos abiertos. Recuperado de: https:// www.datosabiertos.gob.pe//dataset/ingreso-presupuestal-consulta-amigable-ministerio-de-econom\%C3\%ADa-y-finanzas-mef

Reyes-Tagle, G., \& Ospina, L. (9 de octubre de 2020). Recaudando Bienestar. Amnistías tributarias en tiempos de covid: Medidas excepcionales en tiempos excepcionales. Recuperado de: https://blogs.iadb. org/gestion-fiscal/es/amnistias-tributarias-en-tiempos-de-covid/

Ruiz Vásquez, J. (2017). La cultura tributaria y la gestión municipal. Quipukamayoc, 25(48), 49-60. https://doi. org/10.15381/quipu.v25i48.13992 